Advances in Dynamical Systems and Applications (ADSA).

ISSN 0973-5321, Volume 14, Number 2, (2019). 257-265

(C) Research India Publications

https://dx.doi.org/10.37622/ADSA/14.2.2019.257-265

\title{
An Application of Generalized Laplace Transform in PDEs
}

\author{
Hwajoon Kim ${ }^{1}$ \\ Kyungdong University, 11458, Kyungdong Univ. Rd. 27, Yangju, Gyeonggi, S. Korea. \\ ${ }^{2}$ Supaknaree Sattaso \\ Kasetsart University, Sakon Nakhon 47000, Thailand. \\ ${ }^{3}$ Karuna Kaewnimit, ${ }^{4}$ Kamsing Nonlaopon \\ Khon Kaen University, Khon Kaen 40002, Thailand.
}

\begin{abstract}
We would like to consider an application of generalized Laplace transform in partial differential equations(PDEs) by using the $n$-th partial derivatives. The tool of this research is the induction, and the proposed idea gives an easy solution to engineering problems by freely selecting the integer $\alpha$ in the definition.
\end{abstract}

AMS Subject Classification: 34A25, 44A99, 35A22

Key Words and Phrases: generalized Laplace transform, PDEs, induction

\section{Introduction}

The purpose of this research is to consider an application of generalized Laplace transform, G-transform, in PDEs. The G-transform is not new one but a generalized Laplace transform, and we recognize that the basis of this transform is Laplace transform. Nevertheless, this transform is quite plausible due to various applicability. Many theories about integral transforms such as Sumudu, Fourier, Elzaki, double Laplace, and natural transform[1-2] have covered applications in PDEs. 
While these existing researches on integral transforms have not dealt with the theory in an integrated way, this research is meaningful because it is dealt with the integrated way. Normally, Laplace-typed integral transforms have the form of

$$
\int_{0}^{\infty} k(s, t) f(t) d t
$$

and Laplace transform has the kernel $k(s, t)=e^{-s t}$. Since the exponential function $e^{-s t}$ has a nice property converging to 0 when $t$ approaches $\infty$, we normally use the kernel with this exponential function in transform theories. Since Laplace transform can be rewritten as

$$
\int_{0}^{\infty} e^{-\frac{t}{u}} f(t) d t
$$

by $s=1 / u$, the general form of generalized Laplace transform has the form of

$$
u^{\alpha} \int_{0}^{\infty} e^{-\frac{t}{u}} f(t) d t
$$

as a natural extension[4, 9]. In this comprehensive form, the integer value can be suitably selected in various problems. The value $\alpha=0$ corresponds to Laplace transform[6], and it has a strong point in the transforms of derivatives, whereas $\alpha=-2$ has a strong point in the transforms of integrals[7]. Sumudu transform[10] has a value of $\alpha=-1$ and Elzaki one[3,5] has a value of $\alpha=1$ in the above form. The alternate form of integral transforms is

$$
u^{\beta} \int_{0}^{\infty} e^{-t} f(u t) d t
$$

If $\beta=1$, it corresponds to Laplace transform, if $\beta=0$, Sumudu one, and if $\beta=2$, Elzaki one.

In this article, we would like to propose an application of generalized Laplace transform to PDEs, and the obtained results are as the following;

$$
\begin{aligned}
& \text { (1) } G\left[\frac{\partial^{n} f(x, t)}{\partial x^{n}}\right]=\frac{d^{n} F(x, u)}{d x^{n}} \\
& \text { (2) } G\left[\frac{\partial^{n} f(x, t)}{\partial t^{n}}\right]=\frac{F(x, u)}{u^{n}}-u^{\alpha} \sum_{k=1}^{n} \frac{1}{u^{n-k}} \frac{\partial^{k-1} f(x, 0)}{\partial t^{k-1}}
\end{aligned}
$$

where $u=G(f)=F(u)$ and $\partial^{0} / \partial=1$.

Concomitantly, related examples are considered. 


\section{An application of generalized Laplace transform in PDEs}

We would like to prove the next equalities by induction, and these equalities are well applied to several PDEs.

Theorem 1. If a function $f$ is an integrable, then the following equality holds;

(1) $G\left[\frac{\partial^{n} f(x, t)}{\partial x^{n}}\right]=\frac{d^{n} F(x, u)}{d x^{n}}$.

(2) $G\left[\frac{\partial^{n} f(x, t)}{\partial t^{n}}\right]=\frac{F(x, u)}{u^{n}}-u^{\alpha} \sum_{k=1}^{n} \frac{1}{u^{n-k}} \frac{\partial^{k-1} f(x, 0)}{\partial t^{k-1}}$,

where $u=G(f)=F(u)$ and $\partial^{0} / \partial=1$.

Proof. (1) Let us consider the case of $n=1$.

$$
\begin{gathered}
G\left[\frac{\partial f(x, t)}{\partial x}\right]=u^{\alpha} \int_{0}^{\infty} e^{-\frac{t}{u}} \cdot \frac{\partial f}{\partial x} d t \\
=\frac{\partial}{\partial x}\left(u^{\alpha} \int_{0}^{\infty} e^{-\frac{t}{u}} f(t) d t\right)=\frac{\partial}{\partial x} F(x, u)=\frac{d}{d x} F(x, u) .
\end{gathered}
$$

Next, let us assume that

$$
G\left[\frac{\partial^{n} f(x, t)}{\partial x^{n}}\right]=\frac{d^{n} F(x, u)}{d x^{n}}
$$

is valid for some $n$, and we show that

$$
\begin{gathered}
G\left[\frac{\partial^{n+1} f(x, t)}{\partial x^{n+1}}\right]=\frac{d^{n+1} F(x, u)}{d x^{n+1}} ; \\
G\left[\frac{\partial^{n+1} f(x, t)}{\partial x^{n+1}}\right]=u^{\alpha} \int_{0}^{\infty} e^{-\frac{t}{u}} \cdot \frac{\partial^{n+1} f}{\partial x^{n+1}} d t \\
=\frac{\partial}{\partial x}\left[u^{\alpha} \int_{0}^{\infty} e^{-\frac{t}{u}} \cdot \frac{\partial^{n} f}{\partial x^{n}} \cdot d t\right]=\frac{\partial}{\partial x} \cdot \frac{d^{n} F(x, u)}{d x^{n}}=\frac{d^{n+1} F}{d x^{n+1}}
\end{gathered}
$$

for $\partial^{0} / \partial=1$.

(2) First let us consider the case of $n=1$.

$$
\begin{gathered}
G\left[\frac{\partial f(x, t)}{\partial t}\right]=u^{\alpha} \int_{0}^{\infty} e^{-\frac{t}{u}} \cdot \frac{\partial f}{\partial t} \cdot d t \\
=u^{\alpha}\left[\left[e^{-\frac{t}{u}} f(x, t)\right]_{0}^{\infty}+\frac{1}{u} \int_{0}^{\infty} e^{-\frac{t}{u}} f(x, t) d t\right]=-u^{\alpha} f(x, 0)+\frac{1}{u} F(x, u) .
\end{gathered}
$$

This is the same as the value from statement (2). Next, let us suppose that $n=m$ is valid for some $m$. Thus,

$$
G\left[\frac{\partial^{m} f(x, t)}{\partial t^{m}}\right]=\frac{F(x, u)}{u^{m}}-u^{\alpha} \sum_{k=1}^{m} \frac{1}{u^{m-k}} \frac{\partial^{k-1} f(x, 0)}{\partial t^{k-1}}
$$




$$
\begin{aligned}
=\frac{F(x, u)}{u^{m}}-u^{\alpha}\left[\frac{f(x, 0)}{u^{m-1}}\right. & +\frac{1}{u^{m-2}} \frac{\partial f(x, 0)}{\partial t}+\cdots+\frac{1}{u} \frac{\partial^{m-2} f(x, 0)}{\partial t^{m-2}} \\
& \left.+\frac{\partial^{m-1} f(x, 0)}{\partial t^{m-1}}\right]
\end{aligned}
$$

holds for $\partial^{0} / \partial=1$. Let us show that

$$
\begin{gathered}
G\left[\frac{\partial^{m+1} f(x, t)}{\partial t^{m+1}}\right]=\frac{F(x, u)}{u^{m+1}}-u^{\alpha}\left[\frac{f(x, 0)}{u^{m}}+\frac{1}{u^{m-1}} \frac{\partial f(x, 0)}{\partial t}\right. \\
\left.+\cdots+\frac{1}{u} \frac{\partial^{m-1} f(x, 0)}{\partial t^{m-1}}+\frac{\partial^{m} f(x, 0)}{\partial t^{m}}\right] ;
\end{gathered}
$$

By the idea of $n=1$, we have

$$
\begin{gathered}
G\left[\frac{\partial^{m+1} f(x, t)}{\partial t^{m+1}}\right]=\frac{\partial}{\partial t} G\left[\frac{\partial^{m} f(x, t)}{\partial t^{m}}\right]=\frac{1}{u} \cdot(*)-u^{\alpha} \frac{\partial^{m} f(x, 0)}{\partial t^{m}} \\
=\frac{F(x, u)}{u^{m+1}}-u^{\alpha}\left[\frac{f(x, 0)}{u^{m}}+\frac{1}{u^{m-1}} \frac{\partial f(x, 0)}{\partial t}\right. \\
\left.+\cdots+\frac{1}{u} \frac{\partial^{m-1} f(x, 0)}{\partial t^{m-1}}\right]-u^{\alpha} \frac{\partial^{m} f(x, 0)}{\partial t^{m}} \\
=(* *) .
\end{gathered}
$$

Hence, the theorem is valid in an arbitrary natural number $k$.

Of course, the statement (2) can be rewritten as

$$
G\left[\frac{\partial^{n} f(x, t)}{\partial t^{n}}\right]=\frac{F(x, u)}{u^{n}}-u^{\alpha} \sum_{k=1}^{n} \frac{f^{(k-1)}(x, 0)}{u^{n-k}}
$$

for $f^{(n)}$ is the $n$-th derivative of $f$. Hence, we can use the following equalities in various application;

$$
\begin{gathered}
G\left[\frac{\partial f(x, t)}{\partial t}\right]=\frac{1}{u} G(f)-f(x, 0) u^{\alpha} . \\
G\left[\frac{\partial^{2} f(x, t)}{\partial t^{2}}\right]=\frac{1}{u^{2}} G(f)-\frac{1}{u} f(x, 0) u^{\alpha}-f^{\prime}(x, 0) u^{\alpha} \\
=\frac{1}{u^{2}} G(f)-u^{\alpha}\left[\frac{1}{u} f(x, 0)+f^{\prime}(x, 0)\right] . \\
G\left[\frac{\partial^{n} f(x, t)}{\partial t^{n}}\right]=\frac{1}{u^{n}} G(f)-\frac{1}{u^{n-1}} f(x, 0) u^{\alpha}-\frac{1}{u^{n-2}} f^{(1)}(x, 0) u^{\alpha}-\cdots \\
-\frac{1}{u} f^{(n-2)}(x, 0) u^{\alpha}-f^{(n-1)}(x, 0) u^{\alpha} \\
=\frac{1}{u^{n}} G(f)-u^{\alpha}\left[\frac{1}{u^{n-1}} f(x, 0)+\frac{1}{u^{n-2}} f^{(1)}(x, 0)+\cdots\right. \\
\left.+\frac{1}{u} f^{(n-2)}(x, 0)+f^{(n-1)}(x, 0)\right]
\end{gathered}
$$

for $F(u)=G(f)$. 
Lemma 2. (Lagrange's method[8]) Lagrange's method states that a particular solution $y_{p}$ of $y^{\prime \prime}+p(x) y^{\prime}+q(x) y=r(x)$ on open interval $I$ is

$$
y_{p}(x)=-y_{1} \int \frac{y_{2} r}{W} d x+y_{2} \int \frac{y_{1} r}{W} d x
$$

where $y_{1}, y_{2}$ form a basis of solutions of the corresponding homogeneous equation $y^{\prime \prime}+p(x) y^{\prime}+q(x) y=0$ on $I$, and $W$ is the Wronskian of $y_{1}, y_{2}$.

Corollary 3. A solution of $w_{t t}=c^{2} G\left(w_{x x}\right)$ subject to the conditions $w(0, t)=0$, $w(L, t)=0, w(x, 0)=f(x)$, and $w_{t}(x, 0)=g(x)$ can be represented by $w(x, t)=$ $G^{-1}[F(x, u)]$, where

$$
F(x, u)=A(u)\left(e^{x / c u}-e^{-x / c u}\right)-\frac{u^{\alpha+1}}{c^{2} u^{2}-1} \int[f(x)+u g(x)] d x .
$$

Proof. Taking G-transform on both sides, we have

$$
\frac{1}{u^{2}} F-u^{\alpha}\left[\frac{1}{u} w(x, 0)+w^{\prime}(x, 0)\right]=c^{2} \frac{d^{2} F}{d x^{2}}
$$

for $F(x, u)=G((w(x, t))$. Organizing this equality, we have

$$
\frac{d^{2} F}{d x^{2}}-\frac{1}{c^{2} u^{2}} F=-\frac{u^{\alpha}}{c^{2}}\left[\frac{1}{u} f(x)+g(x)\right] .
$$

Note that a solution of the homogeneous equation

$$
\frac{d^{2} F}{d x^{2}}-\frac{1}{c^{2} u^{2}} F=0
$$

is

$$
F_{h}=A(u) e^{x / c u}+B(u) e^{-x / c u} .
$$

Next, let us find a particular solution of $(* * *)$ by the method of variation of parameters. Since the Wronskian of $e^{x / c u}$ and $e^{-x / c u}$ is $W=-2 / c u$, a particular solution of (***) can be represented as

$$
\begin{aligned}
F_{p} & =-e^{x / c u} \int e^{-x / c u} \cdot \frac{-\frac{u^{\alpha}}{c^{2}}\left[\frac{1}{u} f(x)+g(x)\right]}{-2 / c u} d x \\
& +e^{-x / c u} \int e^{x / c u} \cdot \frac{-\frac{u^{\alpha}}{c^{2}}\left[\frac{1}{u} f(x)+g(x)\right]}{-2 / c u} d x \\
& =-\frac{u}{2 c} u^{\alpha} e^{x / c u} \int e^{-x / c u}\left(\frac{1}{u} f(x)+g(x)\right) d x
\end{aligned}
$$




$$
+\frac{u}{2 c} u^{\alpha} e^{-x / c u} \int e^{x / c u}\left(\frac{1}{u} f(x)+g(x)\right) d x .
$$

From $F(0, u)=G(w(0, t))=G(0)=0$, we have $B(u)=-A(u)$. Hence,

$$
\begin{gathered}
F(x, u)=A(u)\left(e^{x / c u}-e^{-x / c u}\right) \\
-u^{\alpha} \frac{u}{2 c}\left[e^{x / c u} \int e^{-x / c u}\left(\frac{1}{u} f(x)+g(x)\right) d x\right. \\
\left.-e^{-x / c u} \int e^{x / c u}\left(\frac{1}{u} f(x)+g(x)\right) d x\right] .
\end{gathered}
$$

Let us put

$$
I_{1}=e^{x / c u} \int e^{-x / c u}\left(\frac{1}{u} f(x)+g(x)\right) d x
$$

and

$$
I_{2}=e^{-x / c u} \int e^{x / c u}\left(\frac{1}{u} f(x)+g(x)\right) d x .
$$

By the integration by parts, we have

$$
\begin{gathered}
I_{1}=c u e^{x / c u} \int e^{-x / c u}\left(\frac{1}{u} f(x)+g(x)\right) d x-\int c u\left(\frac{1}{u} f(x)+g(x)\right) d x \\
=c u I_{1}-c \int(f(x)+u g(x)) d x
\end{gathered}
$$

and so

$$
(c u-1) I_{1}=c \int(f(x)+u g(x)) d x .
$$

Thus

$$
I_{1}=\frac{c}{c u-1} \int[f(x)+u g(x)] d x
$$

Similarly, we have

$$
I_{2}=\frac{c}{c u+1} \int[f(x)+u g(x)] d x
$$

Therefore, we have

$$
F(x, u)=A(u)\left(e^{x / c u}-e^{-x / c u}\right)-u^{\alpha} \frac{u}{2 c}\left(I_{1}-I_{2}\right) .
$$

Since

$$
\begin{aligned}
I_{1}-I_{2}= & c\left(\frac{1}{c u-1}-\frac{1}{c u+1}\right) \int[f(x)+u g(x)] d x \\
& =\frac{2 c}{c^{2} u^{2}-1} \int[f(x)+u g(x)] d x,
\end{aligned}
$$

we have

$$
F(x, u)=A(u)\left(e^{x / c u}-e^{-x / c u}\right)-\frac{u^{\alpha+1}}{c^{2} u^{2}-1} \int[f(x)+u g(x)] d x .
$$

Taking the inverse transform, we obtain the solution $w(x, t)$. 
Let us see a familiar example. Note that the motion of the string depends on its initial deflection $f(x)$ and initial velocity $g(x)$.

Example 1. Let us consider an elastic string which is fastened at the ends $x=0$ and $x=\pi$, and assume that its initial deflection $w(x, 0)$ and initial velocity $w_{t}(x, 0)$ are both 0 . Then the equation has the form of

$$
w_{t t}=c^{2} w_{x x}
$$

subject to $w(x, 0)=0, w_{t}(x, 0)=0$, and

$$
\lim _{x \rightarrow \infty} w(x, t)=0
$$

and let us assume that the left end of the string is moved in a single sine wave $w(0, t)=f(t)=\sin 2 t$ if $0 \leq t \leq \pi$, and otherwise $w(0, t)=0$.

Solution. Taking G-transform on both sides, we have

$$
\frac{F}{u^{2}}=c^{2} \frac{d^{2} F}{d x^{2}}
$$

because of initial conditions. Organizing the equality, we have

$$
F^{\prime \prime}-\frac{1}{c^{2} u^{2}} F=0 .
$$

Hence,

$$
F(x, u)=A(u) e^{x / c u}+B(u) e^{-x / c u} .
$$

Since $F(0, u)=G(w(0, t))=G(f(t))=F(u)$ and

$$
\begin{gathered}
\lim _{x \rightarrow \infty} F(x, u)=\lim _{x \rightarrow \infty} G(w(x, t))=G\left(\lim _{x \rightarrow \infty} w(x, t)\right)=G(0)=0, \\
F(x, u)=B(u) e^{-x / c u}=F(u) e^{-x / c u}
\end{gathered}
$$

because of $A(u)=0$. Since

$$
G[f(t-a) h(t-a)]=e^{-a / u} F(u)
$$

for $h(t-a)$ is Heaviside function,

$$
w(x, t)=w\left(t-\frac{x}{c}\right) h\left(t-\frac{x}{c}\right)=\sin 2\left(t-\frac{x}{c}\right)
$$

if $x / c \leq t \leq x / c+\pi$.

Conflict of interest The author declare that there is no conflict of interest regarding the publication of this paper.

Acknowledgements This research was supported by Kyungdong University Research Grant(2019). 
Table 1: Table of Laplace-typed integral transform $G_{\alpha}$ [4]

\begin{tabular}{ccc}
\hline & $\mathrm{f}(\mathrm{t})$ & $G_{\alpha}(f)$ \\
\hline 1 & 1 & $u^{\alpha+1}$ \\
1 & $\mathrm{t}$ & $u^{\alpha+2}$ \\
3 & $t^{n}$ & $n ! \cdot u^{n+\alpha+1}$ \\
4 & $e^{a t}$ & $\frac{u^{\alpha+1}}{1-a u}$ \\
5 & $\sin a t$ & $\frac{a u^{\alpha+2}}{1+u^{2} a^{2}}$ \\
6 & $\cos a t$ & $\frac{u^{\alpha+1}}{1+u^{2} a^{2}}$ \\
7 & $\sinh a t$ & $\frac{a u^{\alpha+2}}{1-u^{2} a^{2}}$ \\
8 & $\cosh a t$ & $\frac{u^{\alpha+1}}{1-u^{2} a^{2}}$ \\
9 & $e^{a t} \cos b t$ & $\frac{u^{\alpha}\left(\frac{1}{u}-a\right)}{\left(\frac{1}{u}-a\right)^{2}+b^{2}}$ \\
10 & $e^{a t} \sin b t$ & $\frac{b u^{\alpha}}{\left(\frac{1}{u}-a\right)^{2}+b^{2}}$ \\
\hline
\end{tabular}

\section{References}

[1] S. A. P. Ahmadi, Hosseinzadeh, H., and Cherati, A.Y., A New Integral Transform for Solving Higher Order Linear Ordinary Laguerre and Hermite Differential Equations, International Journal of Applied and Computational Mathematics, 5 (2019), https://doi.org/10.1007/s40819-019-0712-1

[2] F. B. M. Belgacem and S. Sivasundaram, New developments in computational techniques and transform theory applications to nonlinear fractional and stochastic differential equations and systems, Nonlinear Studies, 22 (2015), 561-563.

[3] T. M. Elzaki, S. M. Ezaki and E. M. A. Hilal, ELzaki and Sumudu Transform for Solving some Differential Equations, Glob. J. of Pure \& Appl. Math., 8 (2012), 167-173.

[4] $\mathrm{Hj} . \mathrm{Kim}$, The intrinsic structure and properties of Laplace-typed integral transforms, Mathematical Problem in Engineering, 2017 (2017), 1-8.

[5] Hj. Kim, The time shifting theorem and the convolution for Elzaki transform, Int. J. of Pure \& Appl. Math. 87 (2013), 261-271.

[6] Hj. Kim, The solution of the heat equation without boundary conditions, Dynamic Systems and Applications, 27 (2018), 653-662.

[7] $\mathrm{Hj}$. Kim, On the form and properties of an integral transform with strength in integral transforms, Far East. J. Math. Sci. 102 (2017), 2831-2844. 
[8] E. Kreyszig, Advanced Engineering Mathematics, Wiley, Singapore, 2013.

[9] S. Supaknaree, K. Nonlaopon, and Hj. Kim, Further properties of Laplace-type integral transforms, Dynamic Systems and Applications, 28 (2019), 195-215.

[10] G. K. Watugula, Sumudu Transform: a new integral transform to solve differential equations and control engineering problems, Int. J. of Math. Edu. in Sci. \& Tech., 24 (1993), 409-421. 